\title{
Wirkungsaspekte literarischer Suiziddarstellungen Essay
}

\section{Thoughts on Impacts of Suicide Stories}

\author{
Arno Herberth
}

\begin{abstract}
Media influence on human behaviour is a fascinating public health topic. The impact of media reporting suicides on suicidal behaviour is of outmost importance and is documented by several studies in social sciences.

This link between actual acts of suicide and literature seems to be a thing of the past. Nevertheless, analysing the plots of stories can contribute to a better understanding of effects a story might have on its audience. On the one hand, the construction of a plot can lead to immersion into a story world. In this case, copy-cat-behaviour could be enhanced under specific circumstances. On the other hand, the aesthetics of a plot can underpin the coincidence of depicted life events and thus avoid simple explanations.

A plot-analysis that takes an alternative plot-development into account could also contribute to a suicide-preventive analysis of literature like the Papageno-Effect, an inverted Werther-Effect that implies less suicides after reporting the mastery of suicidal crisis.

Literary works that do not present simple explanations for suicides may support a better understanding of suicides in real life and can contribute a destigmatized attitude towards suicidal behaviour.
\end{abstract}

\section{Keywords}

suicide, emplotment, impact of suicide stories, Papageno effect 


\section{Suizid und Ansteckung}

Von dem Gedanken, dass individuelle menschliche Handlungen von medialen Darstellungen beeinflusst oder angestoßen werden, geht ein gewisses Faszinosum aus. Neuere Studien lassen einen Zusammenhang beispielsweise zwischen Rauchen in Filmen und tatsächlichem Tabakkonsum unter Jugendlichen plausibel erscheinen. ${ }^{1}$ In gesundheitspolitischer Hinsicht besonders relevant ist der Umstand, dass auch filmische oder printmediale Darstellungen des Suizids Selbsttötungen bei bereits vulnerablen, suizidgefährdeten Menschen auslösen können (= Werther-Effekt). ${ }^{2}$ Vorauszusetzen ist dabei, dass es sich bei dem betreffenden Medium um ein breit rezipiertes Leitmedium handelt, das ein größeres Publikum erreicht. Bis zur Breitenwirksamkeit des Internets war dies ohne Zweifel noch die Tageszeitung und das Fernsehen. Als neues Supramedium, das sowohl für die ehemaligen Leitmedien Zeitung und Fernsehen Darstellungsmodi bietet als auch durch Foren und soziale Medien ganz neue Kommunikationsformen ermöglicht, kann das Web 2.0 gelten. Gerade für die Suizidprävention eröffnet sich damit ein neues Feld der Forschung in Verbindung mit neuen Interventionsmöglichkeiten. ${ }^{3}$

Literarische Werke werden heutzutage nicht mehr in einen ursächlichen Zusammenhang mit Suizidraten gebracht. Dies mag einerseits darin begründet sein, dass die Literatur nicht mehr als das Leitmedium gilt, wie das noch für die zweite Hälfte des 18. Jahrhunderts und teilweise für das 19. Jahrhundert der Fall war. Andererseits exponieren literarische Werke ihre Inhalte auf andere Weise, als dies beispielsweise Zeitungen oder Internetseiten machen. In der Regel muss man sich den Inhalt eines Romans oder einer Erzählung in intensiver Lektüre erschließen. Die hier zum Einsatz kommende Muße mag mit einer unreflektierten Übernahme von Rollenmodellen, wie sie für die medial induzierten Nachahmungssuizide angenommen wird, nicht in Einklang gebracht werden können.

Für den historischen Fall des sogenannten Werther-Fieber, im Rahmen dessen es sogar zu einer Suizid-Epidemie als Folge der Werther-Lektüre gekommen sein mag, kann ein solch erheblicher Anstieg der Suizide nicht bestätigt werden. ${ }^{4}$ Wenngleich die Datenlage für die Werther-Zeit dürftig ist, so sind dennoch nur vereinzelte Fälle in Textzeugnissen belegt, die einen ursächlichen Zusammenhang zwischen Lektüre und individuellem Suizid suggerieren. Auf textstruktureller Ebene kann man im Falle des Werther

1 Vgl. z.B. WAYLEN, Andrea [et al.]: Cross-sectional association between smoking depictions in films and adolescent tobacco use nested in a British cohort study. In: thorax 66, 2011, S. 856-861. https://thorax.bmj. com/content/thoraxjnl/66/10/856.full.pdf

2 Vgl. ETZERSDORFER, Elmar [et al.]: Newspaper reports and suicide. In: The New England Journal of Medicine, 327, 1992, S. 502-503. https://www.nejm.org/doi/full/10.1056/NEJM199208133270720 und SCHMIDTKE, Armin und HÄFNER, Heinz: Die Vermittlung von Selbstmordmotivation und Selbstmordhandlung durch fiktive Modelle. Die Folgen der Fernsehserie „Tod eines Schülers“. In: Nervenarzt 57, 1986 , S. $502-510$.

3 Siehe z.B. TILL, Benedikt und NIEDERKROTENTHALER, Thomas: Surfing for suicide methods and help: Content analysis of websites retrieved with search engines in Austria and the United States. In: The Journal of Clinical Psychiatry, 75, 2014, S. 886-892.

4 Vgl. NIEDERKROTENTHALER, Thomas; HERBERTH, Arno und SONNECK, Gernot: Der Werther-Effekt: Mythos oder Realität? In: Neuropsychiatrie, 21, 2007, S. 284-290. 
eine differenzierte Schilderung der Motivlage, die ständigen Fokalisierungswechsel des Briefromans und eine vorurteilsfreie Behandlung der Thematik als Textmerkmale ins Treffen führen, die einem Nachahmungseffekt zuwiderlaufen würden. Allerdings stellt sich die Frage in prinzipieller Hinsicht, inwieweit das Arrangement von Ereignissen auf einer Plotlinie nicht doch einer Komplexitätsreduktion zuarbeitet, die die Suggestivkraft von Literatur erhöht und die Übernahme von Rollenmodellen, wie sie durch literarische Figuren repräsentiert werden, begünstigt.

\section{Emplotment als Komplexitätsreduktion?}

Plot als Begriff in der Narratologie kann zwischen den analytischen Ebenen story und discourse angesiedelt werden. Die story stellt gleichsam das abstrakte Substrat einer Geschichte dar, wohingegen der Begriff des discourse die Art und Weise der Realisierung einer Erzählung in ihrer Verlaufsform bezeichnet. Mit Emplotment kann das Nacheinander-Reihen von Ereignissen und Geschehnissen auf einer Zeitachse verstanden werden, wodurch ein kausales Verhältnis zwischen diesen Ereignissen bzw. Geschehnissen gestiftet wird. So betrachtet scheint der Begriff des Plots der discourse-Ebene näher zu sein, allerdings fließen in die Beschreibung bzw. Konstitution eines Plots Hypothesen bzw. Feststellungen zum Warum des jeweils konkreten Plotarrangements mit ein. Es gibt also hier in der Analyse resp. Rezeption einen Dialog zwischen den Ebenen der story und des discourse. ${ }^{5}$ Dieser Dialog kann dann noch an Komplexität zunehmen, wenn nicht realisierte Plot-Stränge, die sich nur angedeutet oder in Träumen bzw. Phantasien realisiert wiederfinden, in die Analyse miteinbezogen werden. ${ }^{6}$

Die Hypothese, dass vor allem als trivial einzustufende Erzählformen einem einfachen Plot-Schema folgen, das Erwartungshaltungen einer LeserInnenschaft bedient und angesichts kontingenter sozialer Realitäten einer Komplexitätsreduktion dienlich ist, mag sich leicht bestätigen lassen. Für komplexer strukturierte Erzählwerke, die eine gleichermaßen komplizierte Plotform aufweisen, wird diese Annahme jedoch nicht haltbar sein. Plot-Arrangements können die LeserInnen auch in die Irre führen, die Kontingenz von Ereignissen betonen, anstatt diese mit einfachen Kausalitäten zu erklären oder ein Eintauchen in erzählte Welten gerade dadurch verhindern, indem das Emplotment in selbstreflexiven Passagen, die über die Erzählkonstruktion Auskunft geben, erläutert wird.

Letzteres ist beispielsweise sowohl in Unterm Rad von Hermann Hesse als auch in Der Schüler Gerber von Friedrich Torberg in ironischer Absicht der Fall. Gerade durch diese Ironisierung erfahren die LeserInnen eine Distanzierung von den Figuren und deren Verstrickungen in einen Handlungsverlauf, laufen also nicht mit den Figuren auf einen finalen Punkt hin zu (etwa den Suizid als finales Schlussereignis eines Erzähltextes), sondern bekommen auf subtile Weise eine Außenperspektive präsentiert, die einerseits

5 Vgl. dazu KUKKONEN, Karin: Plot, Paragraphe 1. In: HÜHN, Peter [et al.] (Hg.): the living handbook of narratology. Hamburg 2014. http://www.lhn.uni-hamburg.de/article/plot

6 Vgl. dazu den Ansatz von RYAN, Marie-Laue: Possible Worlds, Artificial Intelligence, and Narrative Theory. Indiana 1996, S. 116. 
Identifikation behindert und ihnen andererseits bewusst macht, dass es sich um ein Stück Fiktion handelt, das eigenen Konstruktionsmechanismen folgt. ${ }^{7}$ Die Betrachtung des Emplotments stellt ein wesentliches textanalytisches Instrumentarium dar, das zur Beschreibung von Wirkungspotentialen literarischer Texte herangezogen werden kann.

\section{Papageno-Effekte literarischer Suiziddarstellungen?}

Wie die Arbeiten von Thomas Niederkrotenthaler nahelegen, gibt es einen sich zum Werther-Effekt gegengleich verhaltenden Effekt, der in der Fachliteratur mit dem Terminus Papageno-Effekt bezeichnet wird. Wird die Bewältigung einer suizidalen Krise in printmedialen Berichten geschildert oder angedeutet, so konnten im unmittelbaren Zeitraum nach der Publikation des betreffenden Artikels geringere Suizidraten beobachtet werden als im Vergleichszeitraum. Ebenso waren Berichte, in denen Suizidphantasien, jedoch keine Suizidversuche oder Suizide thematisiert wurden, mit einer niedrigeren Suizidrate assoziiert. ${ }^{8}$

Relevant sind solche Fragestellungen, wie sie etwa in Niederkrotenthalers Studie von 2010 gestellt werden, vor allem für Print- und andere Massenmedien. Allerdings ist es verlockend, zumindest im Sinne eines Gedankenexperiments, die aus dieser Studie gewonnenen Erkenntnisse hypothetisch auf die Literatur zu übertragen bzw. den Erkenntnishorizont hier noch wesentlich zu erweitern.

$\mathrm{Zu}$ fragen wäre einerseits, inwieweit bzw. wie prominent alternative Plotverläufe in Suiziderzählungen mit dem Suizid als Schlussereignis angedeutet werden. Gerade dann, wenn hier - trotz letalem Schlusspunkt - ein Möglichkeitsspielraum in der Verlaufsform einer Erzählung etabliert wäre, ließe sich die Frage stellen, ob ein gleichermaßen starker Sog für die LeserInnen entsteht, wenn der Plot Abbiegeoptionen zur tatsächlichen Finalisierung der Narration enthält.

Andererseits stehen Erzählungen jeglichen Inhalts in einem kulturellen Kontext und sind mithin ein Barometer dafür, was in einer bestimmten Zeitepoche überhaupt thematisierbar resp. sagbar ist. In einer Langzeitperspektive ist es sicher günstiger, wenn Erfahrungen mit Suizidalität auch artikuliert werden können. Zum einen ist es für Nahestehende von SuizidentInnen wesentlich, eine Ansprache im Hinblick auf das Erlebte bekommen zu können. Zum anderen ist es für jeden einzelnen essentiell, etwaige Suizidgedanken ausdrücken zu können, ohne dafür stigmatisiert zu werden. Eine Gesellschaft, die zumindest solche Räume - seien diese therapeutischer oder amikaler Natur - schafft, in denen Suizidgedanken geäußert werden können, schafft damit gleichzeitig auch die

7 Vgl. dazu HERBERTH, Arno: Der Jugendsuizid in der Moderne. Wissenschaftliche Vermessung und literarischer Diskurs. Wien 2014, Kapitel 7 und 9. http://othes.univie.ac.at/34083/1/2014-05-26_9806621.pdf

8 Vgl. NIEDERKROTENTHALER, Thomas [et al.]: Role of media reports in completed and prevented suicide: Werther v. Papageno effects. In: British Journal of Psychiatry. 197, 2010, S. 234-243. https://s3.amazonaws.com/academia.edu.documents/42021360/Role_of_media_reports_in_completed_and_p2016020318384-65lp5e.pdf?AWSAccessKeyId=AKIAIWOWYYGZ2Y53UL3A\&Expires $=1533983192 \&$ Signature $=$ yoGFUpuEMsctDrSyVLLfZ\%2BCh4DA\%3D\&response-content-disposition=inline\%3B\%20filename\%3DRole_of_media_reports_in_completed_and_p.pdf 
Möglichkeit, Hilfe und Unterstützung anbieten zu können. Inwieweit hier die Literatur ein solches gesellschaftliches Klima, in dem über Suizid gesprochen werden kann, mit erzeugt, ist hier die relevante kulturwissenschaftliche Fragestellung, die sowohl für einzelne Texte vor dem Hintergrund eines historisch zu fundierenden Suiziddiskurses gestellt wird als auch in Bezug auf größere Textkorpora oder in komparatistischer Hinsicht ergiebige Ergebnisse erzielen kann.

Für das 18. Jahrhundert mag es unbestritten der Fall sein, dass hier der Suiziddiskurs eine Erweiterung durch die Literatur widerfahren hat, da singuläre literarische Werke wie Goethes Werther oder Christian Heinrich Spieß’ Biographien der Selbstmörder dezidiert auch mit dem Anspruch aufgetreten sind, auf den gesellschaftlichen Umgang mit SuizidentInnen verändernd zu wirken. Den Einfluss der Literatur auf den Umgang mit Suizidalität in der Gegenwart einzuschätzen, mag sich als wesentlich komplexer erweisen, da aufgrund der fehlenden zeitlichen Distanz die tatsächliche Wirksamkeit historischer Suiziddiskurse erst entsprechend beurteilt werden muss.

Literarische Werke können jedenfalls einen Beitrag dazu leisten, Suizide und Suizidalität ein Stück weit besser zu verstehen, sofern sie der psycho-sozialen Komplexität suizidaler Prozesse auch gerecht werden. ${ }^{9}$ Sie haben damit einen unmittelbaren gesellschaftlichen Effekt, der allerdings nicht von Fragen der Ästhetik entkoppelt zu betrachten ist, wie die Überlegungen zur Plotkonstruktion gezeigt haben.

\section{Literaturverzeichnis}

ETZERSDORFER, Elmar [et al.] (1992): Newspaper reports and suicide. In: The New England Journal of Medicine, 327, S. 502-503. https://www.nejm.org/doi/full/10.1056/ NEJM199208133270720

HERBERTH, Arno (2014): Der Jugendsuizid in der Moderne. Wissenschaftliche Vermessung und literarischer Diskurs. Wien. http://othes.univie.ac.at/34083/1/2014-05-26_9806621.pdf

KUKKONEN, Karin (2014): Plot, Paragraphe 1. In: Hühn, Peter [et al.] (Hg.): the living handbook of narratology. Hamburg. http://www.lhn.uni-hamburg.de/article/plot

NIEDERKROTENTHALER, Thomas [et al.] (2010): Role of media reports in completed and prevented suicide: Werther v. Papageno effects. In: British Journal of Psychiatry. 197, S. 234-243. https://s3.amazonaws.com/academia.edu.documents/42021360/Role_of_media_reports_in_ completed_and_p20160203-18384-65lp5e.pdf?AWSAccessKeyId=AKIAIWOWYYGZ2Y53UL3A\&Expires=1533983192\&Signature=yoGFUpuEMsctDrSyVLLfZ\%2BCh4DA\%3D\&response-content-disposition=inline\%3B\%20filename\%3DRole_of_media_reports_in_completed_ and_p.pdf

RYAN, Marie-Laue (1996): Possible Worlds, Artificial Intelligence, and Narrative Theory. Indiana. SCHMIDTKE, Armin und HÄFNER, Heinz (1986): Die Vermittlung von Selbstmordmotivation und Selbstmordhandlung durch fiktive Modelle. Die Folgen der Fernsehserie „Tod eines Schülers“. In: Nervenarzt 57, S. 502-510. 
TILL, Benedikt und NIEDERKROTENTHALER, Thomas (2014): Surfing for suicide methods and help: Content analysis of websites retrieved with search engines in Austria and the United States. In: The Journal of Clinical Psychiatry, 75, S. 886-892.

WAYLEN, Andrea [et al.] (2011): Cross-sectional association between smoking depictions in films and adolescent tobacco use nested in a British cohort study. In: thorax 66, S. 856-861. https:// thorax.bmj.com/content/thoraxjnl/66/10/856.full.pdf

Mag. Dr. Arno Herberth / arno.herberth@univie.ac.at

Universität Wien, Fachbereich Neuere Deutsche Literatur,

Universitätsring 1, 1010 Wien, AT 